\title{
An extension of the information-deductive analysis of form'
}

JOHN B. THURMOND AND EARL A. ALLUISI

UNIVERSITY OF LOUISVILLE

Twenty-four Ss responded in a paper-and-pencil figurecancellation task to 4-by-4 metric histoforms and polygons of both random and constrained types. Perceptual performance (number of figures correctly cancelled per minute) was better with random than constrained figures of both types, but the constraint was less disturbing with polygons than with histoforms. Over-all performance was the same with histoforms and polygons.

When man perceives form, he identifies a specific shape as belonging to one of a number of classes, e.g., a table or a chair. Thus, form perception is an information-reduction process that should be inversely related to the uncertainty of the stimulus: the lower the uncertainty, the more likely is the form to be perceived rapidly and accurately.

This approach has been supported by several studies. For example, response time has been found to increase with increasing stimulus uncertainty (or figure complexity) and with use of a constrained (Redundancy-I) sampling rule rather than a random one (Baker \& Alluisi, 1962). Other kinds of redundancy have been found to improve performance, especially in the filtering of noisy displays (Rappaport, 1957). Accuracy has been found to be a positively accelerated decreasing function of the percentage of visual noise perturbing the figures (Alluisi et al, 1964). These studies, however, were conducted with a single class of figuresthe relatively simple "metric histoforms" that look like solidly contoured bar graphs (Baker \& Alluisi, 1962).

These studies are extended here to include "metric polygons" that look like the polygons described by Attneave \& Arnoult (1956). Specifically, the effects of using constrained (Redundancy-I) figures are measured with both histoforms and polygons. Although changes in this transphenomenal parameter affect only the uncertainty of the figures and not their shapes, it has produced consistently detrimental effects on the perception of histoforms (Alluisi \& Hall, 1965; Anderson \& Leonard, 1958; Baker \& Alluisi, 1962). Method

Four-column metric histoforms were sampled from the 256 random and the 24 possible constrained figures in the population. A random and a constrained 8-column metric histoform is shown in Fig. 1-A and 1-C, respectively. Note that each possible column height appears once and only once in the constrained figure. Random and constrained metric polygons were constructed from a "circular matrix" of points in which radii and points are analogs of the columns and cells of the histoform's square matrix; this is shown in Fig. 1-B and 1-D.

The metric histoforms were constructed by typing directly onto spirit masters solid squares (1/12-in. high) produced by a special key on an electric typewriter. The metric polygons were accurately drawn on graph paper, and transferred onto heat-sensitive spirit masters. Both types of figures appeared as solidly dark forms on one side of 8-1/2-by-11-in. sheets of white paper. The areas of the square and the circular matrices were equal.

A sample of 24 random metric histoforms was drawn at random to serve as target figures in one condition, and the 24 possible constrained histoforms were used as targets in a second. Third and fourth conditions were constructed by substituting the polygon analog for each metric histoform.

The 24 target figures of a given type appeared on each of four different sheets-two sheets of random figures and two of constrained. Each sheet consisted of 24 problems arranged in two columns of 12 problems, the columns' being separated by two heavy lines drawn

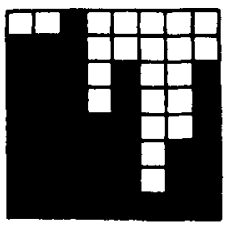

A

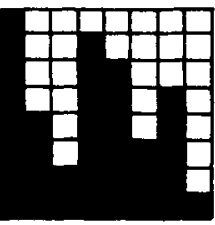

C

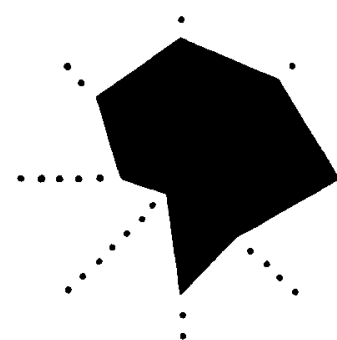

B

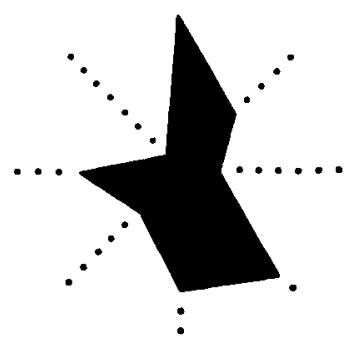

D
Fig. 1. Two different types of matrices and samples of metric figures: (A) random metric histoform, (B) random metric polygon (C) constrained metric histoform, (D) constrained metric polygon. 
down the center of the page. Each problem consisted of a target figure on the left, and three choice figures on the right. The first and second choice figures were metric figures of the same type as the target, whereas the third choice figure was an open square equal in area to the metric histoform cell matrix (1/3-in. high). The task required $S$ to look at the target figure on the left of each row, and then to cross out either the first or the second choice figure if it was identical to the target, or the open square if neither of the choice figures matched the target.

The two sheets of a given kind of target (histoform or polygon) and condition (random or constrained) formed a subtest, and the four appropriate subtests were stapled together to form a test. The order of pages was balanced within subtests, the order of subtests was balanced within each figure-type condition, and the order of figure-type condition was balanced across tests.

The tests were administered to 24 psychology students (16 males and 8 females) at the University of Louisville; Ss ranged in age from 18 to 39 years, with a median of 20 .

Elapsed time from the beginning of testing was written in 5-sec. intervals on a blackboard in front of the group-testing room by $E$; the digits were large and clearly visible to all Ss. Each $S$ recorded this time at the bottom of each page as it was completed. Thus, each of the 24 completed test booklets consisted of four cancellation responses (two each with metric histoforms and metric polygons) for each of the 48 target figures (24 random and 24 constrained), or 192 responses in all, and also of eight elapsed time recordings-one on each of the eight sheets of the test booklet.

\section{Resulis}

Preliminary inspection of the mean time per response and the mean percentage of errors revealed an obvious trade-off between speed and errors, so the two criteria were combined into a single measure. The mean number of correct responses per minute was 28.95 and 22.42 with the random and constrained histoforms, respectively; it was 27.62 and 22.34 with the random and constrained polygons.

An analysis of variance of the data was computed on the basis of the 3-dimensional factorial design of the study, with the dimensions identified as subjects (S), type of figure (T), and sampling rule (R). The results of the analysis indicated that more random figures were identified correctly per minute than constrained figures $(F=126.170, d f=1 / 23, p<.01)$. The $T-b y-R$ interaction was also significant $(F=5.698$, $\mathrm{df}=1 / 23, \mathrm{p}<.05$ ), with the constraining rule's being relatively less disturbing to the polygons than to the histoforms. The difference between the figure types (metric histoforms and polygons) did not reach statistical significance $(F=1.012, d f=1 / 23, p>.25)$.

\section{Discussion}

The circular matrix used to construct metric polygons is entirely permissible in an informational-deductive analysis of form, and each polygon has an analog in a metric histoform generated from a square matrix. Its use permits the variation and control of stimulus uncertainty to be extended to metric polygons as well as metric histoforms. There are two additional advantages: first, the number of angles in the polygon's contour is equal to the number of points used in its construction. Secondly, identical polygons are not generated in different locations; this avoids a major obstacle to the quantification of Attneave-Arnoult (1956) figures.

The improved performance in identifying polygons with the use of random rather than constrained figures in the present study agrees with previous findings using metric histoforms. On the other hand, the failure to find a difference between the two in overall performance indicates that the same perceptual laws apply to both metric histoforms and polygons, at least with respect to the effects of the transphenomenal variable studied.

\section{References}

Alluisi, E. A., \& Hall, T. J. Effects of a transphenomenal parameter on the visual perception of form. Psychon. Sci., 1965, 3 , 543-544.

Alluisi, E. A., Hawkes, G. R., \& Hall, T. J. Effect of distortion on the identification of visual forms under two levels of multipletask performance. J. engng. Psychol., 1964, 3, 29-40.

Anderson, N. S., \& Leonard, J. A. The recognition, naming, and reconstruction of visual figures as a function of contour redundancy. J. exp. Psychol., 1958, 56, 262-270.

Atneave, F., \& Amoult, M. D. The quantitative study of shape and pattern perception. Psychol. Bull., 1956, 53, 452-471.

Baker, E. J., \& Alluisi, E. A. Information handling aspects of visual and auditory form perception. J. engng. Psychol., 1962, 1, 159-179.

Rappaport, M. The role of redundancy in the discrimination of visual form. J. exp. Psychol., 1957, 53, 3-10. Note

1. Supported in part by the U. S. Army Medical Research and Development Command, Department of the Army, under Research Contract No. DA-49-193-MD-2567, "Behavioral Effects of Infectious Diseases." 